\title{
Robust Stabilization of Nonlinear Systems with Uncertain Varying Control Coefficient
}

\author{
Zaiyue Yang, ${ }^{1}$ C. W. Chan, ${ }^{2}$ and Yiwen Wang ${ }^{3}$ \\ ${ }^{1}$ State Key Laboratory of Industrial Control Technology, Institute of Industrial Process Control, Zhejiang University, Hangzhou, China \\ ${ }^{2}$ Department of Mechanical Engineering, University of Hong Kong, Hong Kong \\ ${ }^{3}$ Qiushi Academy for Advanced Studies, Zhejiang University, Hangzhou, China
}

Correspondence should be addressed to Yiwen Wang; eewangyw@zju.edu.cn

Received 10 December 2013; Accepted 23 March 2014; Published 14 April 2014

Academic Editor: Rongni Yang

Copyright (C) 2014 Zaiyue Yang et al. This is an open access article distributed under the Creative Commons Attribution License, which permits unrestricted use, distribution, and reproduction in any medium, provided the original work is properly cited.

\begin{abstract}
This paper investigates the stabilization problem for a class of nonlinear systems, whose control coefficient is uncertain and varies continuously in value and sign. The study emphasizes the development of a robust control that consists of a modified Nussbaum function to tackle the uncertain varying control coefficient. By such a method, the finite-time escape phenomenon has been prevented when the control coefficient is crossing zero and varying its sign. The proposed control guarantees the asymptotic stabilization of the system and boundedness of all closed-loop signals. The control performance is illustrated by a numerical simulation.
\end{abstract}

\section{Introduction}

The control design for nonlinear uncertain systems has been the research focus in the community for decades [1-3]. In recent years, the problem of uncertain control coefficient has attracted increasing research interests [4-17]. This special type of uncertainty is vital to control performance, because the control coefficient represents the system motion direction under any control, and unsuccessful controllers may lead to positive feedback and instability. Several methods have been proposed in literature to handle this problem $[5,7,9,10]$. Among these studies, an adaptive function was proposed by Nussbaum [9] for linear time-invariant systems to deal with the uncertain but constant control coefficient. Now, the Nussbaum function has already become a standard technique targeting the uncertain control coefficient for both linear and nonlinear systems $[18,19]$. In order to complete more complicated control tasks, the Nussbaum function has also been combined with other control techniques, such as robust control $[8,12]$, adaptive control [15-17], learning control [6, $11]$, and backstepping design $[8,15,16]$.

However, most previous results only investigate a relatively simple case that the sign of the uncertain control coefficient is fixed, that is, either positive or negative. It is because of the fact that the conventional Nussbaum function requires the control coefficient to be sign-fixed or "bounded away from zero." Unfortunately, the general case of signvarying uncertain control coefficient has received much less attention and has not been fully solved yet. Undoubtedly, this problem is technically more challenging and cannot be directly handled by the conventional Nussbaum function. In particular, to design a successful controller, two critical issues have to be taken into full consideration. First, the sign may vary very rapidly and be difficult to track. Second, any control will lose its power when the control coefficient is crossing zero; that is, singular points of control exist and improper controllers probably result in finite-time escape phenomenon [20].

The first attempt in addressing the problem of signvarying uncertain control coefficient for a scalar nonlinear system was reported in [7]. Instead of using the Nussbaum function, an online estimator of the control coefficient was used in the robust control design. However, in order to launch the online estimation mechanism successfully, several restrictive assumptions are made in their work. For example, 
besides assuming the known bounds of the control coefficient and its varying speed, it also requires the control coefficient to be a common term between the unknown dynamics and the control. These assumptions make their method specific instead of general, and thus they need to be relaxed.

In this paper, we propose a new Nussbaum function, which does not require such assumptions of [7] and is able to track the rapidly varying sign of control coefficient. Then, a robust controller is designed and then combined with the proposed Nussbaum function, such that the potential finitetime escape phenomenon is avoided. By such means, all closed-loop signals are bounded and asymptotic stabilization is achieved. The paper is organized as follows. The control problem is presented in Section 2. Section 3 designs the new Nussbaum function and the robust controller, followed by the convergence analysis in Section 4. The illustrative example is given in Section 5.

\section{System Description}

In order to highlight the development of the proposed control approach, we will only consider the following scalar uncertain nonlinear system in this paper:

$$
\dot{x}(t)=f(x)+a(x, t) u,
$$

where $t \in[0,+\infty)$ is the time, $x \in R$ is the state, and $u \in R$ is the control. $f(x)$ is an uncertain continuous function, which denotes the dynamic part of the system and is assumed to be bounded by a known function $\rho(x)$ as follows:

$$
|f(x)| \leq \rho(x)
$$

where $\rho(x)>0$ is well-defined in the sense that $\rho(x)$ is finite for any finite $x$.

$a(x, t)$ is an uncertain continuous function that denotes the sign-varying uncertain control coefficient. In this paper, let the control coefficient $a$ consist of two terms as follows:

$$
a(x, t)=b(x, t) s(x, t),
$$

where $b(x, t)$ and $s(x, t)$ are both unknown continuous functions and govern, separately, the "amplitude" and "sign" of $a$. Let $b(x, t)$ be a bounded positive function satisfying

$$
b(x, t) \in\left[b_{\text {low }}, b_{\text {up }}\right]>0,
$$

where $b_{\text {low }}$ and $b_{\text {up }}$ are unknown positive constants. Without loss of any generality, let the "sign" function $s(k)$ be bounded by 1 as follows:

$$
|s(x, t)| \leq 1
$$

Thus, we immediately have

$$
|a(x, t)| \leq b_{\text {up }}
$$

in previous literature $[8,9,15,16]$.

However, in this paper $s(x, t)$ is allowed to vary its value and change its sign. Compared with most previous results, this study is more comprehensive and technically challenging. The focus of research will be put on handling the varying $s(x, t)$, which has been barely addressed. Since $s(x, t)$ continuously varies between positive and negative and will certainly cross zero, let the control singular point be denoted by the pair $\left(x_{i}, t_{i}\right) \in R \times[0,+\infty), i=1,2, \ldots$, such that,

$$
s\left(x_{i}, t_{i}\right)=0 .
$$

Note that at these points, the system is essentially out of control for any $u$; thus, the dynamics are solely determined by $f(x)$. Therefore, in general, the control singular points are considered to be separately located, so that the control $u$ only loses its power at these particular points; otherwise, finite-escape phenomenon may be produced by $f(x)$ when $u$ remains useless for a period of $s(x, t)=0$. According to this consideration, the following assumption is made, which in fact is quite weak and can be easily satisfied for most common continuous functions.

Assumption 1. Assume that there always exists an arbitrarily small positive constant $\delta$, such that one and only one control singular point locates in the neighborhood $|s(x, t)| \leq \delta$, while $|s(x, t)| \geq \delta$ outside these neighborhoods.

In addition, since $f(x)$ may not stabilize the system at the origin point $x=0$, it has to require that the control $u$ be effective when $x=0$, or equivalently, $s(0, t)$ is a nonzero constant instead of a control singular point for any $t$. In other words, $s(x, t)$ keeps varying between positive and negative when $x \neq 0$ and stops until $x=0$. To characterize this point, another assumption is imposed.

Assumption 2. Assume that the derivative of $s(x, t)$ can be written into

$$
\frac{d s}{d t}=c(x, t) h(x)
$$

where $h(x)$ is a known function satisfying

$$
h(x) \begin{cases}\neq 0 & \text { when } x \neq 0 \\ =0 & \text { when } x=0\end{cases}
$$

Thus, $d s / d t=0$ when $x=0$ for any $t . c(x, t)$ stands for the "speed" of sign variation and is an unknown bounded positive function as follows:

$$
c(x, t) \in\left[c_{\text {low }}, c_{\text {up }}\right]>0,
$$

where $c_{\text {low }}$ and $c_{\text {up }}$ are unknown positive constants.

Remark 3. Though $s(0, t)$ is a nonzero constant when $x=0$, $b(0, t)$ as well as $a(0, t)$ is not necessarily a constant and may still vary.

Remark 4. Though $h(x)$ needs to be known for control design in the next section, the other functions, that is, $a(x, t), b(x, t)$, $s(x, t)$, and $c(x, t)$, as well as their bounds, that is, $b_{\text {low }}, b_{\text {up }}, c_{\text {low }}$, $c_{\text {up }}$, and $\delta$, are completely unknown. Therefore, it still provides us great flexibilities to model the control coefficient and to admit various kinds of uncertainties. 
Remark 5. As stated before, the focus of this research is to tackle the continuously varying "sign" function $s(x, t)$. Note that since the unknown function $c(x, t)$ could be very large, $s(x, t)$ may vary very rapidly between positive and negative, which undoubtedly increases the difficulty to track the varying sign. In addition, because there exist some control singular points where the system is essentially out of control, the controller must be carefully designed to avoid the potential finite-time escape phenomenon.

\section{Controller Design}

Let us define the Lyapunov function $V(x)$ such that

$$
\begin{gathered}
V(x) \begin{cases}>0 & \text { when } x \neq 0 \\
=0 & \text { when } x=0,\end{cases} \\
\frac{d V}{d x} \triangleq D(x)=\operatorname{sgn}(x) \frac{|h(x)|}{\rho(x)} .
\end{gathered}
$$

Since $|h(x)| \geq 0$ and $\rho(x)>0$ are known functions, one can always solve $V(x)$ by using the differential equation (12) and $V(0)=0$ as initial condition. By using (12) to define a Lyapunov function, the information of $h(x)$ can be introduced into the controller design process; thus, it is able to avoid the finite-time escape phenomenon at control singular points as shown later. Consequently, the dynamics of $V(x)$ can be expressed as follows:

$$
\dot{V}=\frac{d V}{d x} \dot{x}=D(x)(f(x)+a u) .
$$

Now, design the control $u$ as follows:

$$
u=g(\theta) z
$$

Conceptually, $z$ is a common robust controller [21] that can stabilize the system $\dot{x}(t)=f(x)+z$, that is, the simplified form of (1) when $a=1$, and $g(\theta)$ is the Nussbaum function that is used to deal with the uncertain and varying $a$.

Rewrite (13) as follows:

$$
\dot{V}=D(x)(f(x)+z)+D(x)(a u-z),
$$

and let $z$ be given as follows:

$$
z=-\frac{D(x) \rho^{2}(x)}{|D(x)| \rho(x)+e^{-t}}=-\frac{\operatorname{sgn}(x)|h(x)| \rho(x)}{|h(x)|+e^{-t}} .
$$

Then, one can readily have

$$
\begin{aligned}
D(x)(f(x)+z) & \leq|D(x) f(x)|+D(x) z \\
& \leq|D(x)| \rho(x)-D(x) \frac{\operatorname{sgn}(x)|h(x)| \rho(x)}{|h(x)|+e^{-t}} \\
& =|h(x)|-\frac{h^{2}(x)}{|h(x)|+e^{-t}} \\
& =\frac{|h(x)| e^{-t}}{|h(x)|+e^{-t}} \leq e^{-t} .
\end{aligned}
$$

Taking (17) into (15) yields

$$
\dot{V} \leq e^{-t}+D(x)(a u-z)=e^{-t}+(a g(\theta)-1) D(x) z .
$$

Now, we will design the Nussbaum function $g(\theta)$, which can adjust its value between positive and negative according to the control performance index $\theta$. In this paper, $\theta$ is defined as follows:

$$
\dot{\theta}=-D(x) z=\frac{h^{2}(x)}{|h(x)|+e^{-t}}, \quad \theta_{0}=0 .
$$

It readily computes the lower and upper bounds of $\dot{\theta}$ as follows:

$$
\dot{\theta} \in\left[\max \left(0,|h(x)|-e^{-t}\right),|h(x)|\right] \geq 0 .
$$

Then (18) can be rewritten into

$$
\dot{V} \leq(1-a g(\theta)) \dot{\theta}+e^{-t}
$$

and taking integration yields

$$
\begin{aligned}
V_{t} & \leq \int_{0}^{t} \dot{\theta} d \tau-\int_{0}^{t} \operatorname{ag}(\theta) \dot{\theta} d \tau+V_{0}+\int_{0}^{t} e^{-\tau} d \tau \\
& \leq \theta_{t}-\int_{0}^{\theta_{t}} \operatorname{ag}(\theta) d \theta+V_{0}+1,
\end{aligned}
$$

where $V_{t}=V(t), V_{0}=V(0)$, and $\theta_{t}=\theta(t)$.

Conventionally, the Nussbaum gain often adopts the form $g(\theta)=\exp \left(\theta^{2}\right) \cos (\theta), \exp \left(\theta^{2}\right) \sin (\theta), \theta^{2} \cos (\theta)$, or $\theta^{2} \sin (\theta)$, so that $g(\theta)$ can swing between positive infinite and negative infinite according to the control performance index $\theta$. Thus, it provides a possibility of correcting inappropriate deviation caused by erroneous previous control. However, the signvarying speed of the conventional Nussbaum gain is limited. For example, each variation of the $\operatorname{sign}$ of $\exp \left(\theta^{2}\right) \sin (\pi \theta)$ needs $\theta$ to increase 1 ; therefore, it may not be able to track the rapidly varying $s(k)$ in this paper. To deal with this, a new Nussbaum function is proposed as follows:

$$
g(\theta)=2 \theta e^{\theta^{2 \eta}} \sin \left(\pi \theta^{2}\right)
$$

where the constant $\eta>1$ is a design parameter. Note that $\theta^{2}$ grows nonlinearly, and thus the sign-varying speed of $g(\theta)$ keeps increasing with the increase of $\theta$. Consequently, the proposed $g(\theta)$ varies much faster than those conventional ones when $\theta$ is sufficiently large. The property of the proposed $g(\theta)$ is presented below.

Lemma 6. Suppose that $|s(x, t)| \geq \varepsilon$, where $\varepsilon$ is an arbitrary positive constant that is smaller than 1. If $\operatorname{ag}(\theta)>0$ for $\theta^{2} \epsilon$ $\left[\left\lfloor\Theta^{2}\right\rfloor-1,\left\lfloor\Theta^{2}\right\rfloor\right]$ when $\Theta \rightarrow \infty$, then we have

$$
\lim _{\Theta \rightarrow \infty} \sup \frac{1}{\Theta^{2}} \int_{0}^{\Theta} a g(\theta) d \theta=+\infty
$$

where $\lfloor\cdot\rfloor$ is the truncation operator; otherwise, if ag $(\theta)<0$ for $\theta^{2} \in\left[\left\lfloor\Theta^{2}\right\rfloor-1,\left\lfloor\Theta^{2}\right\rfloor\right]$ when $\Theta \rightarrow \infty$, then

$$
\lim _{\Theta \rightarrow \infty} \inf \frac{1}{\Theta^{2}} \int_{0}^{\Theta} a g(\theta) d \theta=-\infty .
$$

The detailed proof of Lemma 6 is given in the Appendix. The property of $g(\theta)$ will be used to derive the asymptotic convergence in the next section. 


\section{Convergence Analysis}

Before presenting the main theorem of asymptotic convergence, the following lemma is introduced first.

Lemma 7. If $\theta_{t}$ is bounded for any $t \in[0,+\infty)$, all closedloop signals are bounded; that is, $\theta_{r}$ and $V_{r}$ are bounded for $r \in[0, t)$, and the system is asymptotically stabilized; that is, $x \rightarrow 0$ when $t \rightarrow+\infty$.

Proof. The proof of Lemma 7 is straightforward. Since $|h(x)|$ is semipositive, from (19), we have

$$
\dot{\theta}=\frac{h^{2}(x)}{\|h(x)\|+e^{-t}} \begin{cases}>0 & \text { when } x \neq 0 \\ =0 & \text { when } x=0 .\end{cases}
$$

Clearly, $\theta$ is a semipositive and nondecreasing variable. Thus the boundedness of $\theta_{t}$ implies the boundedness of $\theta_{r}$ for $r \in[0, t)$. Then according to (22), since $a, g(\theta)$, and $V_{0}$ are all bounded, $V_{r}$ is also bounded when $\theta_{r}$ is bounded. On the other hand, the boundedness of $\theta_{t}$ also implies that $\dot{\theta} \rightarrow 0$ when $t \rightarrow+\infty$, since $\dot{\theta}$ is continuous and semipositive; or equivalently, $|h(x)| \rightarrow 0$ when $t \rightarrow+\infty$. Then from (9), we can readily conclude that $x \rightarrow 0$ when $t \rightarrow+\infty$. This completes the proof.

Now, the main result is stated below.

Theorem 8. The proposed controller in (14), (16), (20), and (23) guarantees the boundedness of all closed-loop signals and is able to drive $x \rightarrow 0$ when $t \rightarrow+\infty$.

Proof. As shown in Lemma 7, the key of the proof is to show the boundedness of $\theta_{t}$. Because $s(x, t)$ may cross zero and change its sign, we will consider two situations: (1) $s(x, t)$ is crossing zero, and (2) $s(x, t)$ is bounded away from zero. Next, we will prove that $\theta_{t}$ is bounded under both situations.

Situation 1. $s(x, t)$ is crossing zero. Let $s\left(x_{i}, t_{i}\right)=0$ and consider a small neighborhood of it; that is, $s(x, t) \mid \leq \varepsilon$, where $\varepsilon=$ $\delta / 2$ is a small positive constant. Since the positive constant $\delta$ in Assumption 1 can be arbitrarily small, it still has only one control singular point in the neighborhood $|s(x, t)| \leq \varepsilon=$ $\delta / 2$. Without loss of any generality, consider the situation that $s$ varies from negative to positive. Let $s=-\varepsilon$ at $t_{-\varepsilon}, s=\varepsilon$ at $t_{\varepsilon}$, and $t_{-\varepsilon}<t_{i}<t_{\varepsilon}$. From (8), one can readily yield

$$
\begin{gathered}
s\left(x, t_{-\varepsilon}\right)=\int_{0}^{t_{-\varepsilon}} c(x, \tau) h(x) d \tau+s_{0}=-\varepsilon, \\
s\left(x, t_{\varepsilon}\right)=\int_{0}^{t_{\varepsilon}} c(x, \tau) h(x) d \tau+s_{0}=\varepsilon .
\end{gathered}
$$

Consequently, we have

$$
\begin{aligned}
\int_{t_{-\varepsilon}}^{t_{\varepsilon}} h(x) d \tau & \leq \frac{\int_{t_{-\varepsilon}}^{t_{\varepsilon}} c(x, \tau) h(x) d \tau}{\min (c(x, t))} \leq \frac{\varepsilon-s_{0}-\left(-\varepsilon-s_{0}\right)}{c_{\text {low }}} \\
& =\frac{2 \varepsilon}{c_{\text {low }}} .
\end{aligned}
$$

Note that since $s$ varies from negative to positive, $d s / d t>0$ for $\tau \in\left[t_{-\varepsilon}, t_{\varepsilon}\right]$, or equivalently, $h(x)>0$ for $\tau \in\left[t_{-\varepsilon}, t_{\varepsilon}\right]$. Then, according to (20), (29) implies that

$$
\theta_{t_{\varepsilon}}-\theta_{t_{-\varepsilon}} \leq \int_{t_{-\varepsilon}}^{t_{\varepsilon}}|h(x)| d \tau=\int_{t_{-\varepsilon}}^{t_{\varepsilon}} h(x) d \tau \leq \frac{2 \varepsilon}{c_{\mathrm{low}}} .
$$

Since $c_{\text {low }}$ is positive constant, $2 \varepsilon / c_{\text {low }}$ is bounded. Equation (30) indicates that the increment of $\theta$ for $\tau \in\left[t_{-\varepsilon}, t_{\varepsilon}\right]$, or equivalently, in the neighborhoods of control singular points, is bounded. Note that (30) also implies that the finite-time escape phenomenon will not occur in these neighborhoods, since finite increment of $\theta$ insures finite increment of $V$ according to (22).

Situation 2. $s(x, t)$ is bounded away from zero. Consider a continuous interval $|s| \geq \varepsilon$ between two successive control singular points. Note that from Assumption 1, the sign of $s$ will not change and $\max (|s|) \geq \delta=2 \varepsilon$ in this interval. Without loss of any generality, let $s=\varepsilon$ at $t_{\varepsilon}$ and $s=\delta=2 \varepsilon$ at $t$. The boundedness of $\theta_{t}$ within this interval will be proven by the contradiction method; that is, we will first assume that $\theta_{t}$ is unbounded and then derive some contradictions.

Since $\theta \geq 0$, if $\theta_{t}$ is unbounded, we have $\theta_{t} \rightarrow+\infty$. As $V_{t} \geq 0,(22)$ gives

$$
0 \leq V_{t} \leq \theta_{t}-\int_{0}^{\theta_{t}} a g(\theta) d \theta+V_{0}+1,
$$

and we further have

$$
\frac{1}{\theta_{t}^{2}} \int_{0}^{\theta_{t}} \operatorname{ag}(\theta) d \theta \leq \frac{1}{\theta_{t}^{2}}+\frac{1}{\theta_{t}}+\frac{V_{0}}{\theta_{t}^{2}},
$$

which implies that

$$
\lim _{\theta_{t} \rightarrow+\infty} \frac{1}{\theta_{t}^{2}} \int_{0}^{\theta_{t}} \operatorname{ag}(\theta) d \theta \leq 0 .
$$

Next, we will deduce a result for $\theta_{t} \rightarrow+\infty$ that contradicts (33), separately, for two cases: (a) $\theta_{\varepsilon}$ is bounded; (b) $\theta_{\varepsilon}$ is unbounded, where $\theta_{\varepsilon}=\theta\left(t_{\varepsilon}\right)$.

(a) $\theta_{\varepsilon}$ Is Bounded. Since $\theta_{t} \rightarrow+\infty$, we conclude that the increment between $\theta_{t}$ and $\theta_{\varepsilon}$ is infinite; that is, $\theta_{t}-\theta_{\varepsilon} \rightarrow+\infty$. It implies that $g(\theta)$ swings infinite times between positive and negative for $\theta \in\left[\theta_{\varepsilon}, \theta_{t}\right]$, or equivalently for $\tau \in\left[t_{\varepsilon}, t\right]$. Since the sign of $s$ is fixed for $\tau \in\left[t_{\varepsilon}, t\right]$, the sign of $a g(\theta)$ is solely determined by $g(\theta)$ for $\tau \in\left[t_{\varepsilon}, t\right]$. Without loss of any generality, let $\operatorname{ag}(\theta)>0$ for $\theta^{2} \in\left[\left\lfloor\theta_{t}^{2}\right\rfloor-1,\left\lfloor\theta_{t}^{2}\right\rfloor\right]$, which satisfies the condition of Lemma 6 . Consequently, according to (24), we have

$$
\lim _{\theta_{t} \rightarrow+\infty} \sup \frac{1}{\theta_{t}^{2}} \int_{0}^{\theta_{t}} a g(\theta) d \theta=+\infty .
$$

Clearly (34) contradicts (33), which implies that $\theta_{t}$ must be bounded if $\theta_{\varepsilon}$ is bounded.

(b) $\theta_{\varepsilon}$ Is Unbounded. Since $\theta \geq 0$, we have $\theta_{\varepsilon} \rightarrow+\infty$. Note that from Assumption 1, the sign of $h(x)$ is fixed for $\tau \in\left[t_{\varepsilon}, t\right]$. 
Without loss of any generality, let $h(x)>0$. Similar to (28), we have

$$
\begin{gathered}
s\left(x, t_{\varepsilon}\right)=\int_{0}^{t_{\varepsilon}} c(x, \tau) h(x) d \tau+s_{0}=\varepsilon, \\
s\left(x, t_{\sigma}\right)=\int_{0}^{t_{\sigma}} c(x, \tau) h(x) d \tau+s_{0}=2 \varepsilon .
\end{gathered}
$$

Then, similar to (30), we further have

$$
\begin{aligned}
\int_{t_{\varepsilon}}^{t} h(x) d \tau & \geq \frac{\int_{t_{\varepsilon}}^{t} c(x, \tau) h(x) d \tau}{\max (c(x, t))} \geq \frac{\int_{t_{\varepsilon}}^{t} c(x, \tau) h(x) d \tau}{c_{\text {up }}} \\
& \geq \frac{\delta-\varepsilon}{c_{\text {up }}}=\frac{\varepsilon}{c_{\text {up }}},
\end{aligned}
$$

which can be simplified as follows:

$$
\int_{t_{\varepsilon}}^{t} h(x) d \tau \geq \gamma
$$

where $\gamma \triangleq \varepsilon / c_{\text {up }}$ is a positive constant since $\varepsilon$ could be an arbitrarily small positive constant.

Then, we will estimate the increment of $\theta_{t}^{2}-\theta_{\varepsilon}^{2}$. Note that $h(x)>0$ for $\tau \in\left[t_{\varepsilon}, t\right]$ and then take integration for (20) as follows:

$$
\begin{aligned}
\theta_{t}-\theta_{\varepsilon} & =\int_{t_{\varepsilon}}^{t} \dot{\theta} d \tau \geq \int_{t_{\varepsilon}}^{t} h(x)-e^{-\tau} d \tau \\
& \geq \int_{t_{\varepsilon}}^{t} h(x) d \tau-\left(e^{-t_{\varepsilon}}-e^{-t}\right)>\gamma-e^{-t_{\varepsilon}} .
\end{aligned}
$$

We can always choose a sufficiently large constant $\bar{t}$, such that for any $t_{\varepsilon} \geq \bar{t}$, we have

$$
e^{-t_{\varepsilon}} \leq \frac{\gamma}{2}
$$

Thus the increment of $\theta_{t}^{2}-\theta_{\varepsilon}^{2}$ for $t_{\varepsilon} \geq \bar{t}$ can be readily estimated by taking (39) into account as follows:

$$
\theta_{t}^{2}-\theta_{\varepsilon}^{2}=\left(\theta_{t}+\theta_{\varepsilon}\right)\left(\theta_{t}-\theta_{\varepsilon}\right)>2 \theta_{\varepsilon}\left(\gamma-\frac{\gamma}{2}\right)=\theta_{\varepsilon} \gamma
$$

Again, note that the sign of $s$ is fixed for $\tau \in\left[t_{\varepsilon}, t\right]$ and the sign of $\operatorname{ag}(\theta)$ is determined by $g(\theta)$. Since $\gamma$ is a positive constant and $\theta_{\varepsilon}$ is unbounded, with the increase of $\theta_{\varepsilon}$, we will certainly have

$$
\theta_{t}^{2}-\theta_{\varepsilon}^{2}>\theta_{\varepsilon} \gamma \geq 3
$$

Clearly, (41) implies that $g(\theta)$ has completed at least a positive round and a negative round when $\theta$ increases from $\theta_{\varepsilon}$ to $\theta_{t}$. Consequently, the conditions of Lemma 6 have been fulfilled. Similar to case (a), we could assume that $a g(\theta)>0$ for $\theta^{2} \epsilon$ $\left[\left\lfloor\theta_{t}^{2}\right\rfloor-1,\left\lfloor\theta_{t}^{2}\right\rfloor\right]$ without loss of any generality. Then according to (24), we have the same result as (34) as follows:

$$
\lim _{\theta_{t} \rightarrow+\infty} \sup \frac{1}{\theta_{t}^{2}} \int_{0}^{\theta_{t}} a g(\theta) d \theta=+\infty,
$$

which still contradicts (33). Therefore, $\theta_{t}$ must be bounded to avoid any contradiction.

In summary, $\theta_{t}$ must be bounded for the intervals of $|s| \geq$ $\varepsilon$ from Situation 2 and its increment is also bounded for the intervals of $|s| \leq \varepsilon$ from Situation 1. As a result, $\theta_{t}$ is always bounded, and thus all closed-loop signals are bounded and $V_{t} \rightarrow 0$ when $t \rightarrow+\infty$. This completes the proof.

Remark 9. Since $\theta_{t}$ is bounded, $\int_{0}^{t} h(x) d \tau$ is also bounded because

$$
\int_{0}^{t} h(x) d \tau \leq \int_{0}^{t} \dot{\theta}+e^{-\tau} d \tau \leq \theta_{t}+1 .
$$

On the other hand, (37) indicates that the integral of $h(x)$ needs to increase at least $\gamma$ to trigger a sign variation of control coefficient. Since $\gamma$ is a positive constant, $\int_{0}^{t} h(x) d \tau / \gamma$ is also bounded, which essentially implies that the control coefficient will only undergo finite number of sign variations in the entire control process.

Remark 10. The proof of the Theorem is organized as follows. First, the finite increment of $\theta$ has been proven in Situation 1 and case (a) of Situation 2, separately, at the control singular points and in the intervals between these points. That is, these two parts show that a complete sign variation process corresponds to finite increment of $\theta$. Second, case (b) of Situation 2 further shows that the control coefficient only has finite number of sign variations. In such a way, the proof of finite $\theta_{t}$ has been completed.

Remark 11. Note that according to Lemma 7, the boundedness of $\theta_{t}$ for $t \in[0,+\infty)$ also implies that no finite-time escape phenomenon will occur, even at the control singular points.

\section{Simulation}

An example is used to illustrate the performance of the proposed control. Consider system (1) with the following setup:

$$
\begin{aligned}
& f(x)=\cos (x) x^{2}, \\
& b(x, t)=\exp (\cos (x)+\sin (t)), \\
& s(k)=\cos (k) \\
& c(x, t)=\exp (\sin (x t)) \\
& h(x)=|x|
\end{aligned}
$$

Let the initial state be $x_{0}=2$ and the known bounding function be $\rho(x)=x^{2}+1$. Note that only $\rho(x)$ and $h(x)$ are known for controller design. Let the controller in (14), (16), (20), and (23) be used with the parameter settings $\eta=1.01$ and $\dot{\theta}=-D(x) z / 10$. It is shown by Figure 1 that the proposed controller can drive $x$ to 0 asymptotically and Figure 2 shows the profile of other bounded variables. 

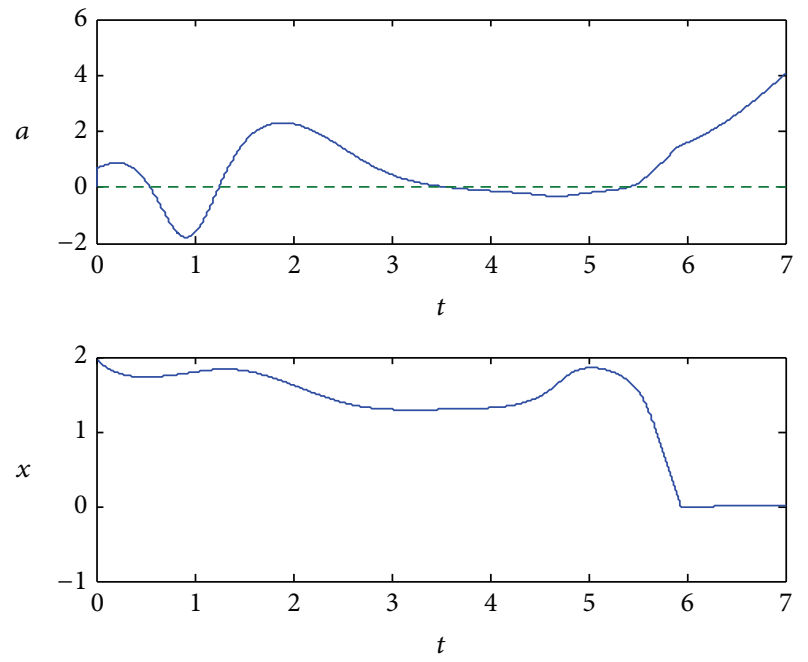

Figure 1: The profiles of $a$ and $x$.
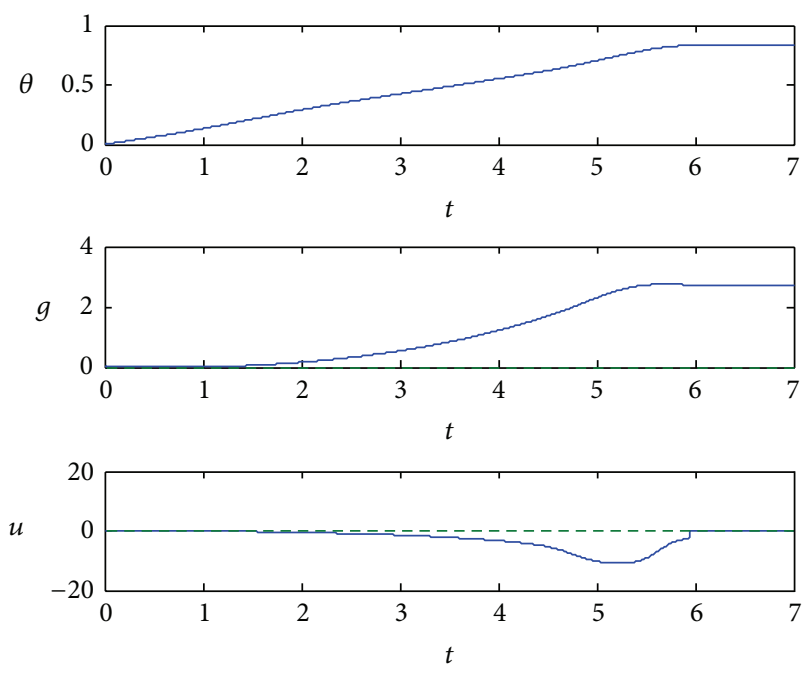

FIGURE 2: The profiles of $\theta, g$, and $u$.

\section{Conclusion}

In this paper, the control problem is studied for a class of nonlinear uncertain systems with the uncertain control coefficient, which is allowed to vary continuously between positive and negative. A new Nussbaum gain is designed and integrated with robust controller to tackle this problem. By following the Lyapunov-fashion controller design procedure, the potential finite-time escape phenomenon is avoided. It is proven that the proposed control approach yields asymptotic stability and guarantees the boundedness of the closed-loop signals.

\section{Appendix}

Proof of Lemma 6. For the concise of the proof, we will only consider the case of $\Theta \rightarrow+\infty$, while the same results can be obtained similarly for $\Theta \rightarrow-\infty$. Now define

$$
G_{i}=\int_{\sqrt{i}}^{\sqrt{i+1}} g(\theta) d \theta,
$$

where $i$ is a positive integer. Further calculation yields

$$
\begin{aligned}
G_{i} & =\int_{\sqrt{i}}^{\sqrt{i+1}} 2 \theta e^{\theta^{2 \eta}} \sin \left(\pi \theta^{2}\right) d \theta=\int_{\sqrt{i}}^{\sqrt{i+1}} e^{\theta^{2 \eta}} \sin \left(\pi \theta^{2}\right) d \theta^{2} \\
& =\int_{i}^{i+1} e^{w^{\eta}} \sin (\pi w) d w
\end{aligned}
$$

where $w=\theta^{2}$. Clearly, $G_{i}$ is positive if $i$ is even and negative if $i$ is odd.

Because $w$ is positive and $\eta>1$, it is readily to show that

$$
(w+1)^{\eta}>w^{\eta}+\eta w^{\eta-1} .
$$

Then, according to (A.3), the ratio between $G_{i+1}$ and $G_{i}$ is

$$
\begin{aligned}
\frac{G_{i+1}}{G_{i}} & =\frac{\int_{i+1}^{i+2} e^{w^{\eta}} \sin (\pi w) d w}{\int_{i}^{i+1} e^{w^{\eta}} \sin (\pi w) d w} \\
& =\frac{\int_{i}^{i+1} e^{(w+1)^{\eta}} \sin (\pi(w+1)) d w}{\int_{i}^{i+1} e^{w^{\eta}} \sin (\pi w) d w} \\
& =\frac{-\int_{i}^{i+1} e^{(w+1)^{\eta}} \sin (\pi w) d w}{\int_{i}^{i+1} e^{w^{\eta}} \sin (\pi w) d w} \\
& <\frac{-\int_{i}^{i+1} e^{w^{\eta}+\eta w^{\eta-1}} \sin (\pi w) d w}{\int_{i}^{i+1} e^{w^{\eta}} \sin (\pi w) d w} \\
& <\frac{-e^{\eta \eta^{i-1}} \int_{i}^{i+1} e^{w^{\eta}} \sin (\pi w) d w}{\int_{i}^{i+1} e^{w^{\eta}} \sin (\pi w) d w} \\
& =-e^{\eta i^{\eta-1}} \cdot
\end{aligned}
$$

Because $\eta i^{\eta-1}>1$ and $e^{\eta i^{\eta-1}}>e$, it is clear that $G_{i} \rightarrow \infty$ and $G_{i+1} / G_{i} \rightarrow-\infty$, when $i \rightarrow+\infty$. Let us investigate the situation of $\Theta^{2}$ being an integer and $\Theta^{2}=\left\lfloor\Theta^{2}\right\rfloor=i+1$; that is, $\operatorname{ag}(\theta)>0$ for $\theta^{2} \in[i, i+1]$. Since $\varepsilon \leq|s(x, t)| \leq 1$, we have $\varepsilon b_{\text {low }} \leq|a| \leq b_{\text {up }}$. Then, we will firstly prove (24). Consider the following:

$$
\begin{aligned}
\int_{0}^{\sqrt{i+1}} \operatorname{ag}(\theta) d \theta & \geq-\int_{0}^{\sqrt{i}}|a g(\theta)| d \theta+\int_{\sqrt{i}}^{\sqrt{i+1}} a g(\theta) d \theta \\
& \geq-b_{\text {up }} \int_{0}^{\sqrt{i}}|g(\theta)| d \theta+\varepsilon b_{\text {low }} \int_{\sqrt{i}}^{\sqrt{i+1}} a g(\theta) d \theta \\
& \geq-b_{\text {up }} \sum_{l=0}^{i-1}\left|G_{l}\right|+\varepsilon b_{\text {low }}\left|G_{i}\right| \\
& \geq-b_{\text {up }}(i-1)\left|G_{i-1}\right|+\varepsilon b_{\text {low }} e^{\eta i^{\eta-1}}\left|G_{i-1}\right| \\
& =\left(-b_{\text {up }}(i-1)+\varepsilon b_{\text {low }} e^{\eta \eta^{i-1}}\right)\left|G_{i-1}\right|
\end{aligned}
$$


Clearly,

$$
\frac{1}{i+1} \int_{0}^{\sqrt{i+1}} \operatorname{ag}(\theta) d \theta \geq\left(-b_{\mathrm{up}} \frac{i-1}{i+1}+\varepsilon b_{\mathrm{low}} \frac{1}{i+1} e^{\eta i^{i-1}}\right)\left|G_{i-1}\right|
$$

Because $b_{\text {low }}, b_{\text {up }}$, and $\varepsilon$ are positive and finite and $\eta>1$, when $i \rightarrow+\infty$, we have

$$
\begin{aligned}
& \lim _{i \rightarrow+\infty}\left(-b_{\text {up }} \frac{i-1}{i+1}+\varepsilon b_{\text {low }} \frac{1}{i+1} e^{\eta i^{\eta-1}}\right)\left|G_{i-1}\right| \\
& =\left(-b_{\text {up }}+\varepsilon b_{\text {low }_{i \rightarrow+\infty}} \lim _{i+1} \frac{1}{i+1} e^{\eta i^{\eta-1}}\right)\left|G_{i-1}\right| \longrightarrow+\infty .
\end{aligned}
$$

Consequently, (A.7) implies that

$$
\lim _{i \rightarrow+\infty} \frac{1}{i+1} \int_{0}^{\sqrt{i+1}} \operatorname{ag}(\theta) d \theta \longrightarrow+\infty
$$

which is the result of $(24)$ since $\Theta^{2}=i+1$.

Similarly, when $|a| \geq \varepsilon b_{\text {low }}$ if $a g(\theta)<0$ for $\theta^{2} \in\left[\left\lfloor\Theta^{2}\right\rfloor-\right.$ $\left.1,\left\lfloor\Theta^{2}\right\rfloor\right]$ with $\Theta^{2}=\left\lfloor\Theta^{2}\right\rfloor=i+1$, we can also show that

$$
\lim _{i \rightarrow+\infty} \frac{1}{i+1} \int_{0}^{\sqrt{i+1}} \operatorname{ag}(\theta) d \theta \longrightarrow-\infty,
$$

which is the result of $(25)$. For the case of $\Theta \rightarrow-\infty$, the same results can also be obtained by similar procedures. The proof is complete.

\section{Conflict of Interests}

The authors declare that there is no conflict of interests regarding the publication of this paper.

\section{Acknowledgments}

This work was supported by the 863 Program under Grant 2012AA041709 and by the NSFC under Grant no. 61004057.

\section{References}

[1] X. Zhang and N. Duan, "Further results on global state feedback stabilization of nonlinear systems with low-order and highorder nonlinearities," International Journal of Innovative Computing, Information and Control, vol. 9, no. 11, pp. 4389-4400, 2013.

[2] H. Wang, B. Chen, and C. Lin, "Adaptive neural tracking control for a class of stochastic nonlinear systems with unknown deadzone," International Journal of Innovative Computing, Information and Control, vol. 9, no. 8, pp. 3257-3269, 2013.

[3] R. Yang, P. Shi, and G.-P. Liu, "Filtering for discrete-time networked nonlinear systems with mixed random delays and packet dropouts," IEEE Transactions on Automatic Control, vol. 56, no. 11, pp. 2655-2660, 2011.

[4] B. D. O. Anderson, S. Dasgupta, and A. C. Tsoi, "On the convergence of a model reference adaptive control algorithm with unknown high frequency gain," Systems and Control Letters, vol. 5, no. 5, pp. 303-307, 1985.
[5] R. Lozano and B. Brogliato, "Adaptive control of a simple nonlinear system without a priori information on the plant parameters," IEEE Transactions on Automatic Control, vol. 37, no. 1, pp. 30-37, 1992.

[6] P. Jiang, H. Chen, and L. C. A. Bamforth, "A universal iterative learning stabilizer for a class of MIMO systems," Automatica, vol. 42, no. 6, pp. 973-981, 2006.

[7] J. Kaloust and Z. Qu, "Robust control design for nonlinear uncertain systems with an unknown time-varying control direction," IEEE Transactions on Automatic Control, vol. 42, no. 3, pp. 393-399, 1997.

[8] L. Liu and J. Huang, "Global robust output regulation of lower triangular systems with unknown control direction," Automatica, vol. 44, no. 5, pp. 1278-1284, 2008.

[9] R. D. Nussbaum, "Some remarks on a conjecture in parameter adaptive control," Systems and Control Letters, vol. 3, no. 5, pp. 243-246, 1983.

[10] T. R. Oliveira, A. J. Peixoto, E. V. L. Nunes, and L. Hsu, "Control of uncertain nonlinear systems with arbitrary relative degree and unknown control direction using sliding modes," International Journal of Adaptive Control and Signal Processing, vol. 21, no. 8-9, pp. 692-707, 2007.

[11] Z. Yang, S. C. P. Yam, L. K. Li, and Y. Wang, "Universal repetitive learning control for nonparametric uncertainty and unknown state-dependent control direction matrix," IEEE Transactions on Automatic Control, vol. 55, no. 7, pp. 1710-1715, 2010.

[12] Z. Yang, S. C. P. Yam, L. K. Li, and Y. Wang, "Robust control for uncertain nonlinear systems with state-dependent control direction," International Journal of Robust and Nonlinear Control, vol. 21, no. 1, pp. 106-118, 2011.

[13] Q. Yang, Z. Yang, and Y. Sun, "Universal neural network control of MIMO uncertain nonlinear systems," IEEE Transactions on Neural Networks \& Learning Systems, vol. 23, no. 7, pp. 11631169, 2012.

[14] Z. Yang, Y. Wang, and Y. Sun, "Universal stabilization design of uncertain nonlinear system with sign-switching unknown control direction," International Journal of Control, vol. 86, no. 1, pp. 172-182, 2013.

[15] X. Ye, "Adaptive nonlinear output-feedback control with unknown high-frequency gain sign," IEEE Transactions on Automatic Control, vol. 46, no. 1, pp. 112-115, 2001.

[16] Y. Xudong and Z. Ding, "Robust tracking control of uncertain nonlinear systems with unknown control directions," Systems and Control Letters, vol. 42, no. 1, pp. 1-10, 2001.

[17] Y. Zhang, C. Wen, and Y. C. Soh, "Adaptive backstepping control design for systems with unknown high-frequency gain," IEEE Transactions on Automatic Control, vol. 45, no. 12, pp. 23502354, 2000.

[18] J. B. D. Cabrera and K. Furuta, "Improving the robustness of Nussbaum-type regulators by the use of $\sigma$-modification-local results," Systems and Control Letters, vol. 12, no. 5, pp. 421-429, 1989.

[19] M. Corless and E. P. Ryan, "Adaptive control of a class of nonlinearly perturbed linear systems of relative degree two," Systems and Control Letters, vol. 21, no. 1, pp. 59-64, 1993.

[20] H. Khalil, Nonlinear Systems, Prentice Hall, 3rd edition, 2001.

[21] Z. Qu, Robust Control of Nonlinear Uncertain Systems, John Wiley \& Sons, New York, NY, USA, 1998. 


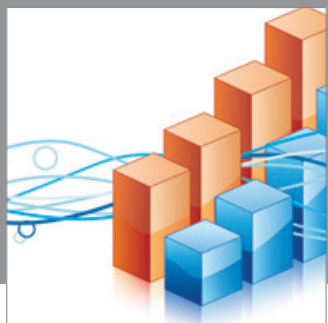

Advances in

Operations Research

mansans

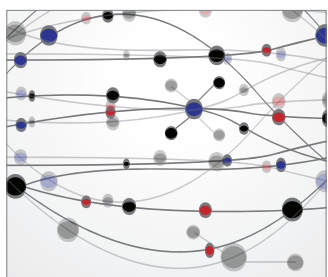

The Scientific World Journal
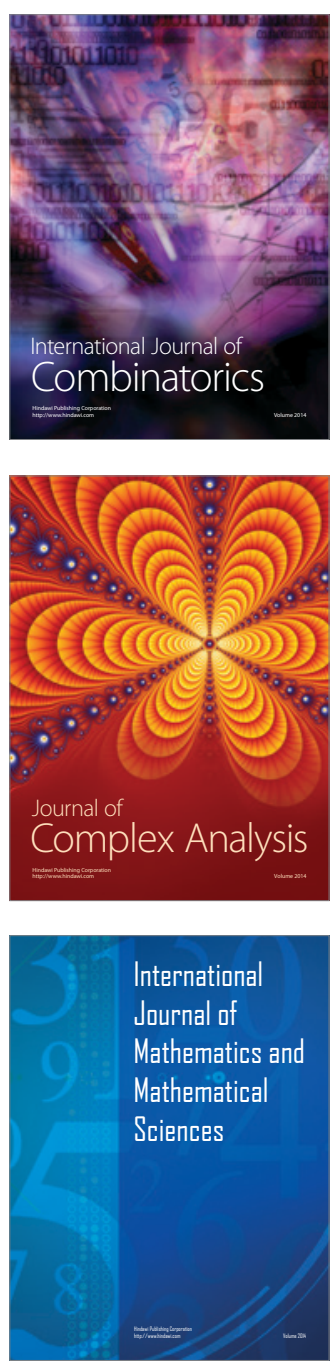
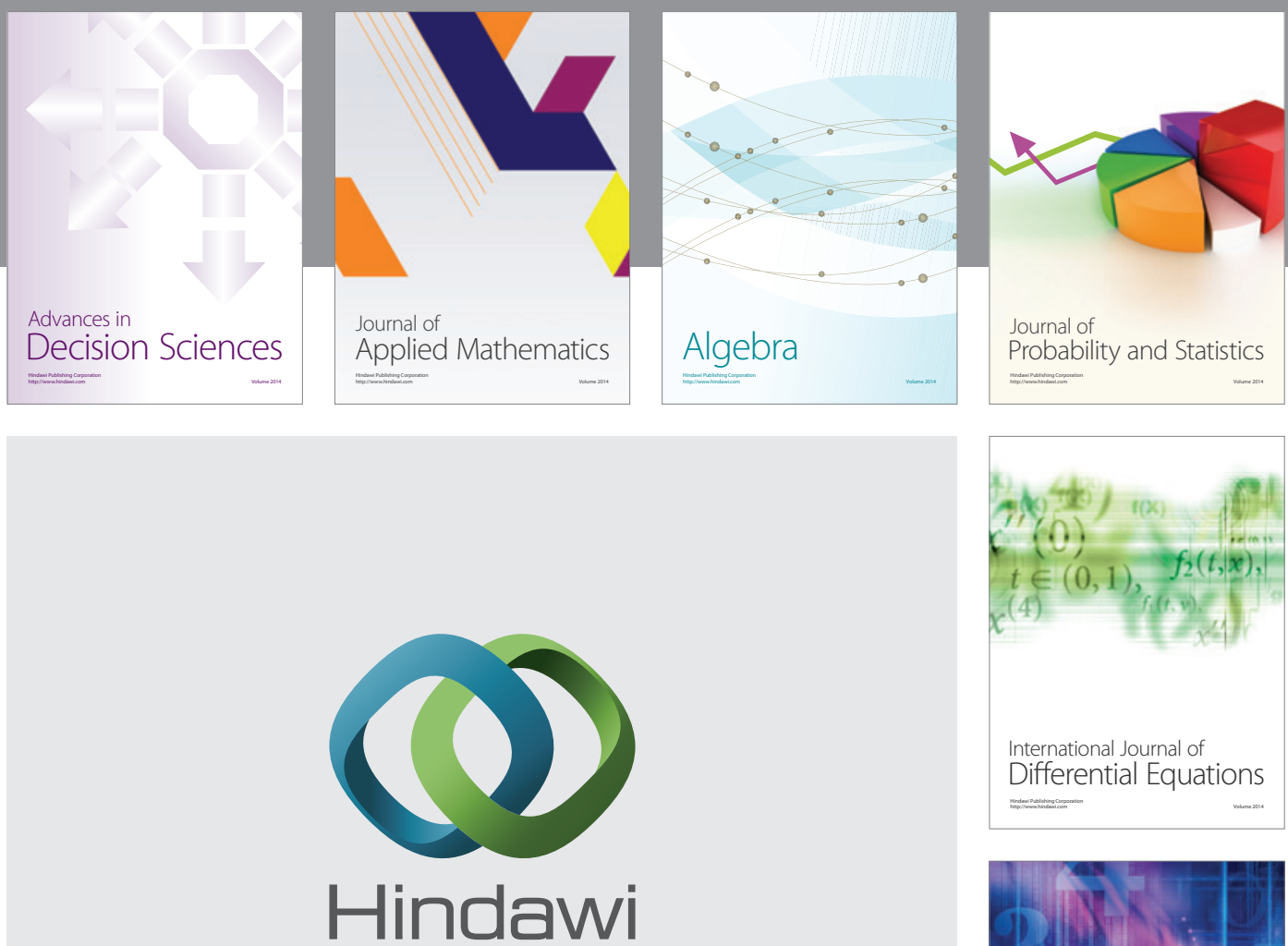

Submit your manuscripts at http://www.hindawi.com
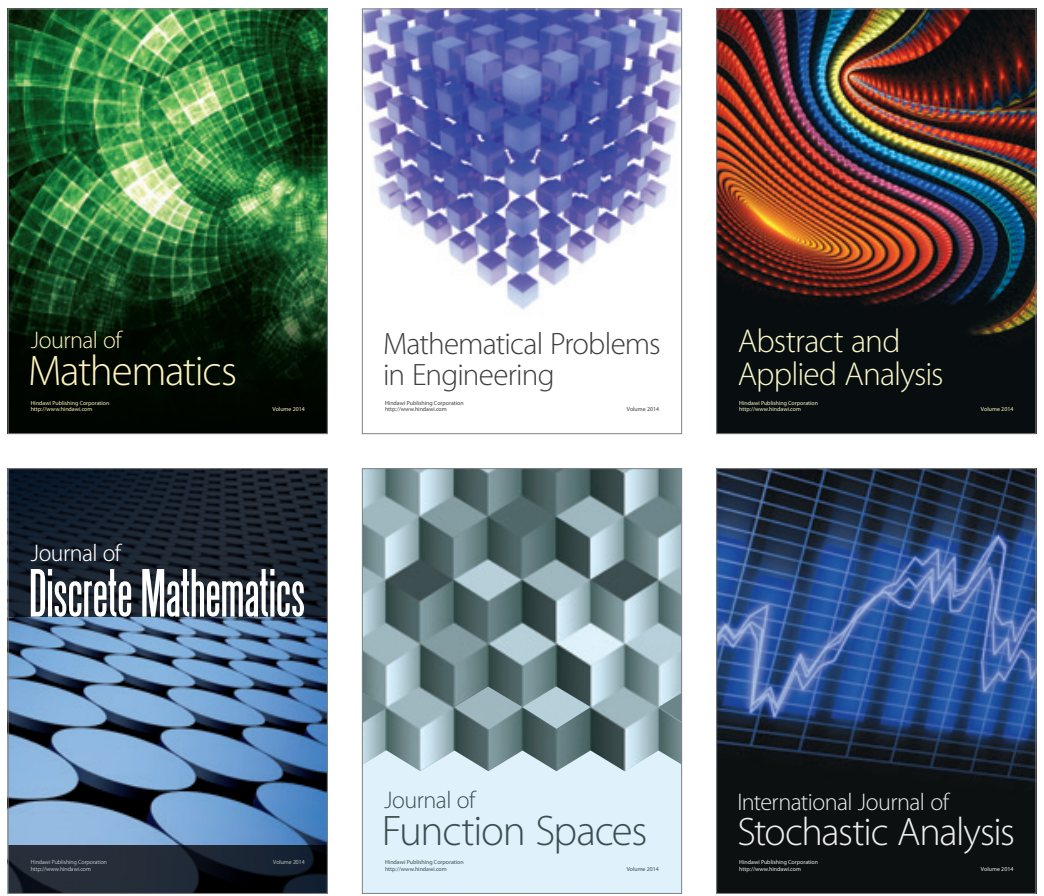

Journal of

Function Spaces

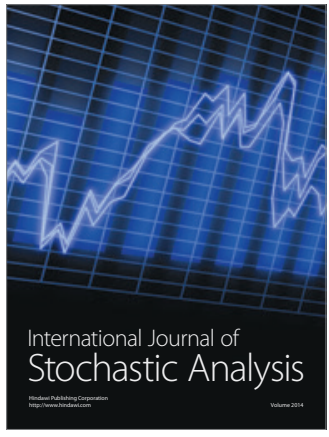

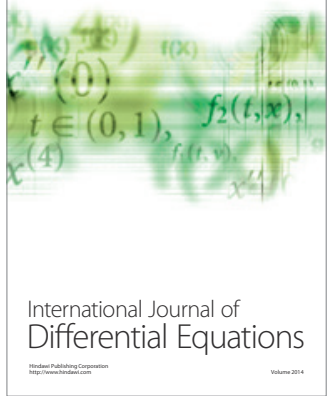
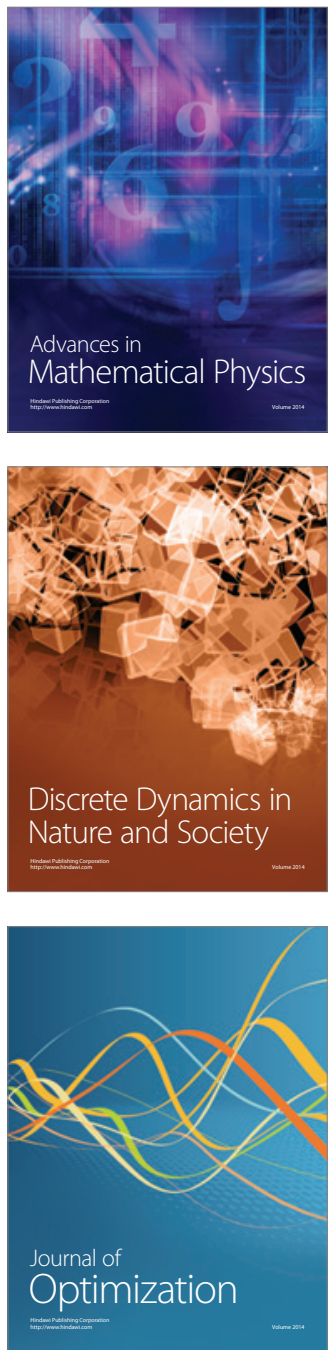